\title{
Complexity in Mathematics and Complexity of a Physical Process
}

\section{Artur Sowa*}

Department of Mathematics \& Statistics, University of Saskatchewan, Canada

The close relationship between mathematics and physical reality is well reflected in one of the fundamental questions of our time: how to assess the complexity of a problem? Whether the problem we consider is in essence mathematical or technological our assessment of its complexity relies upon the same intuition. It is clear that a problem is complex if it is hard to solve. An object is complex if its properties cannot be easily derived from its definition. Also, we expect complexity to be universal and portable, transferrable from one approach to another. In particular, if we know that a problem is computationally hard we will have little hope of solving it easily via a mathematical trick.

Consider the following classical theorem of A.M. Ostrowski [1]:

If a Dirichlet series $D(s)=\alpha_{1}+\alpha_{2} 2^{-s}+\alpha_{3} 3^{-s}+\alpha_{4} 4^{-s}+\ldots$

is a solution of a differential algebraic equation, then the set of all prime factors of all $\mathrm{n}$ for which $\mathrm{a}_{\mathrm{n}} \neq 0$ is finite. This remarkable result implies in particular that the Riemann's zeta function, which is defined via the special Dirichlet series with $a_{n}=1$ for all $n$, is not a solution of any differential algebraic equation. Apparently the zeta function is more complex than any Dirichlet series solution of a differential algebraic equation. The high complexity of the zeta is the very reason it does not admit a simple description such as a differential-algebraic equation would supply. However, loosely speaking, one may view the Dirichlet series whose most or even all $a_{n} \neq 0$ as nontrivial operators in a Hilbert space, [2]. Some of those operators will be related to special bases in the Hilbert space which in turn may, but need not to, stem from a differential algebraic eigenvalue problem. An eigenvalue problem is tantamount to a whole family of differential equations and the complete set of eigen functions is a higher ranking object, or an object of higher complexity, than any single function. While the psychological intuition behind it seems always the same complexity has many avatars, at least one for every particular area of science or culture where it is encountered. In the theory of dynamical systems we take the presence of chaos to be a signature of higher complexity. Likewise, we know that the phenomenon of turbulence is what makes the tasks of predicting the weather or designing airplanes more complex than they would otherwise be. In Computer Science we have the precise notion of algorithm time complexity which relies upon a count of the number of operations required to run it. For comparison, the Complex System Theory, which seeks mathematized concepts and computational methods with which to study the problems of social sciences, often reaches for more intuitive descriptions of complexity such as the dancing landscapes model, [3]. As we know, the discrete Fourier transform was an inefficient computational procedure until Cooley and Tukey found the FFT algorithm. The lesson of this story is quite clear: Despite our a priori intuitions we cannot know for sure whether a given problem is truly complex or not until someone invents an efficient method of solving it or proves that no efficient method can exist. We tend to think that a nut is hard if for a long time many people try to crack it open but to no avail. Yet this is insufficient to conclude that the perceived complexity is real --- perhaps lack of an effective solution at this instance stems from our taking the wrong approach, looking at things from the wrong perspective. One of the famous open questions about the nature of complexity is $\mathrm{P} \neq$ ? NP. Although there have been remarkable results shedding light on its nature, such as the celebrated Razborov-Rudich theorem, [4], we still do not know the answer. Let us briefly return to the signature of complexity that is suggested by the theorem of Ostrowski. Suppose we would like to assess the complexity of a Dirichlet series by computing the prime factors as indicated above. For the complex input such as the series for the zeta function the number of primes at the output will steadily grow, while for a simple input the number of primes on the output will stabilize. An infinite chain of calculations might not be achievable in practice and we will eventually take a guess whether the input is indeed complex or not. However, the main reason that this task is computationally hard is that it involves integer factorization which itself is believed NPhard. This scenario synchronizes rather beautifully with the essential message from the Razborov-Rudich theorem. Namely, one can expect that any computational test of complexity will itself be computationally complex, [5]. Mathematics has always had a close relationship with the contemporaneous technologies. From a certain point of view mathematical proofs are constructions and the notion of construction is essentially physical. In its first advanced stage, reached in antiquity by Euclid, Eratosthenes and Archimedes, mathematics relied on tangible, visible construction. To prove a theorem meant to find a construction with a compass and ruler according to certain rules, and with the support of logical arguments and conventional interpretations. While Calculus was invented by Newton in the age of Restoration in order to describe mechanical systems, in the $20^{\text {th }}$ century the situation was partly reversed as people began using analogue electronic circuits to solve differential equations. When discrete-state machines took over computation they in turn became the technology of mathematics. Although modern day computers mostly bring to mind complex electronics in truth the digital revolution stemmed from the intellectual movements in the early $20^{\text {th }}$ century, particularly the revolution in the philosophy of mathematics initiated perhaps by Bertrand Russell. That culture, as well as the challenges that ensued of the Second World War, brought us a whole slew of intellectual giants, to mention but a few: the logician Gödel, the par excellence applied mathematician von Neumann, the pioneers of theoretical computer science Church and Turing, the Bletchley Park mathematician Newman, and the early Enigma-breaker Rejewski, see e.g. [6]. Those people and their collaborators and scientific kin gradually honed in on the concept of a programmable computing device. Their conceptual advances were luckily concurrent with the development of electronically controlled switches: vacuum tubes ('valves' in British English) and later semiconductor based transistors. Only that remarkable confluence

*Corresponding author: Artur Sowa, Department of Mathematics \& Statistics, University of Saskatchewan, Canada, Tel: 1-306-966-6114; E-mail: sowa@math.usask.ca

Received December 06, 2013; Accepted December 08, 2013; Published December 16, 2013

Citation: Sowa A (2013) Complexity in Mathematics and Complexity of a Physical Process. J Appl Computat Math 2: e134. doi:10.4172/2168-9679.1000e134

Copyright: (c) 2013 Sowa A. This is an open-access article distributed under the terms of the Creative Commons Attribution License, which permits unrestricted use, distribution, and reproduction in any medium, provided the original author and source are credited. 
Citation: Sowa A (2013) Complexity in Mathematics and Complexity of a Physical Process. J Appl Computat Math 2: e134. doi:10.4172/21689679.1000 e134

Page 2 of 2

of conditions and ideas could result in the two famous prototypes of a computer: COLOSSUS and ENIAC. COLOSSUS was built at the Bletchley Park and it is quite easy to see how such a concept could arise from the unique and rich intellectual culture of that place, [7]. ENIAC was physically constructed at the Moore School at the University of Pennsylvania but its conceptual roots spread farther and encompass the Institute for Advanced Studies at Princeton, [8]. The microchip based computing technology and the present day approach to mathematics are joined at the hip just as were the compass-ruler constructions and theoretical geometry in times Hellenistic. With the nascent of quantum computers with prototype devices already being sold while their concept and principle of operation are theoretically debated, [9] it is again a good time to pay close attention to the various nontrivial relations of mathematics and technology. The new technologies may usher in a reevaluation of complexity of some problems, and a revision of the very meaning of complexity. In particular it is now debated whether the paradigm of quantum computing will change our perspective at the complexity of integer factorization, [10]. Applied Mathematics is all about constructions: a mathematician working on an applied problem typically has no choice but to deal with infinities in an approximate way via a constructive and thus intuitionist approach. (It is appropriate to understand the term intuitionism used here in its rigorous philosophical sense introduced by Brouwer, where intuitionism is compared and contrasted with the mathematical Platonism). It is not clear that this provides a full view at the fundamental problems of mathematics but it is quite certain that this activity is never really far from the fundamental and even philosophical aspects of mathematics. Let us look at applied and computational mathematics broadly and open-mindedly, and let us not underestimate its historic or present contributions to the foundations of mathematics.

\section{References}

1. Ostrowski A (1920) Uber Dirichletsche Reihen und algebra ische Differentia gleichungen. Math Zeit 8: 241-298.

2. Sowa $A$ (2013) The Dirichlet ring and unconditional bases in $L 2$ [0,2pi] Functional Analysis and Its Applications 47: 227-232.

3. Page SE (2010) Diversity and Complexity, Princeton University Press

4. Razborov AA, Rudich S (1997) Natural proofs. Journal of Computer and System Sciences 55: 24-35.

5. Chow TY (2011) What is a Natural Proof? Notices of the AMS 58.

6. Copeland BJ (2004) The Essential Turing. The ideas that gave birth to the computer age, Clarendon Press: Oxford.

7. Carter F (2008) Code breaking with the Colossus Computer, Bletchley Park Trust.

8. Edwards JR (2012) A History of Early Computing at Princeton. Turing Centennial Celebration.

9. Mansfield A (2013) NASA buys into 'quantum' computer. BBC News, Science \& Environment.

10. Sowa A (1983) The quantum sieve of Eratosthenes, On Platonism in mathematics. In: Paul Bonacerraf and Hilary Putnam (1983) Philosophy of mathematics, Selected readings, (2nd edn), Cambridge University Press, UK. 